\title{
Generation of Adverse Pressure Gradient in the Circumferential Flashback of a Premixed Flame
}

\author{
Nader Karimi ${ }^{1}$ - Stephen McGrath ${ }^{1}$ - Philip Brown ${ }^{1}$. \\ Johannes Weinkauff ${ }^{2}$. Andreas Dreizler ${ }^{2}$
}

Received: 13 August 2015 / Accepted: 14 December 2015 / Published online: 6 January 2016

(C) The Author(s) 2016. This article is published with open access at Springerlink.com

\begin{abstract}
Circumferential upstream propagation of a premixed flame in a region confined between two concentric tubes is considered. The cold flow in this configuration features rotational motion and the flame is modelled as an interface separating the burned and unburned gases. Through an analytical solution of the integral form of the governing equations, it is shown that the static pressure increases across the flame. Hence, the circumferential propagation of the flame is associated with the generation of an adverse pressure gradient. The theoretical prediction of the pressure increase is, further, supported by the experimental observations and discussed in the context of the theory of flame back pressure. The results extend the recent findings on the generation of adverse pressure gradient during the axial propagation of swirling flames, to the circumferential direction. It is argued that the demonstrated pressure gain across the flame can significantly facilitate flame flashback.
\end{abstract}

Keywords Flame flashback · Premixed swirling flames · Circumferential propagation · Adverse pressure gradient

\section{Introduction}

Achieving major reductions in $\mathrm{CO}_{2}$ emission, simultaneous with low $\mathrm{NO}_{\mathrm{x}}$ and other air pollutants, is a substantial challenge facing the power generation sector $[1,2]$. Utilising lean premixed combustion and replacing conventional fuels with syngas [2,3] or, mixtures of hydrogen and gaseous hydrocarbons [4, 5], are amongst the main means of addressing this challenge. As these mixtures can vary significantly in chemical composition [3], the

Nader Karimi

Nader.Karimi@glasgow.ac.uk

1 School of Engineering, University of Glasgow, Glasgow G12 8QQ, UK

2 Institute of Reactive Flows and Diagnostics, Technische Universität Darmstadt, Darmstadt, Germany 
future combustion systems should be highly fuel flexible [2]. This, in turn, introduces further difficulties $[2,6]$. Variations in fuel properties can cause operational problems such as thermoacoustic instabilities [7] and flame flashback [6]. The latter is, particularly, problematic in premixed combustion of hydrogen containing fuels [8-10]. In principle, the problem is due to the high reactivity of hydrogen and subsequently higher flame speed of hydrogen blends compared to those of conventional fuels [4, 11]. Currently, flame flashback is considered as one of the most significant technical barriers that hinder the development of hydrogen combustion systems [2,6].

Flashback of a premixed flame is usually defined as the undesirable propagation of the reactive front towards the upstream flow. Occurrence of flame flashback can result in the stabilisation of the flame in the upstream section of the burner [10,11]. Flame flashback can, therefore, severely damage the burner and interrupt the operation and hence, should be avoided. Due to the practical significance of this problem, there exists a large number of studies on determining the flashback limits of various burners under different air and fuel conditions, see for example [12-14]. However, investigations of the underlying physics of flame flashback are less frequent. An early physical mechanism of flame flashback was proposed by Lewis and von Elbe [15]. They correlated this phenomenon to the flow retardation in the boundary layer and quenching of the reaction zone by losing heat to the wall. This classical view remained as the only explanation of flame flashback in boundary layers for a long time.

In recent years, the problem of flashback in boundary layer has been revisited by a number of authors [16-21]. Theoretical and experimental studies of Kurdyumov et al. [16, 17] in laminar reactive boundary layers advanced the findings of Lewis and Von Elbe. These authors [16, 17] calculated a critical condition for the flashback of a laminar flame on the basis of asymptotic consideration and single step chemistry. Eicher and Sattelmayer conducted a combined experimental and numerical investigation on a turbulent reactive boundary layer subject to an adverse pressure gradient [18]. They reported substantially different values of the critical velocity gradient compared to those predicted by the theory of Lewis and von Elbe [15]. Eicher and Sattelmayer [18] attributed this distinction to the influence of adverse pressure gradient upon the boundary layer. These authors, further, investigated the wall flashback of methane and hydrogen flames in laminar and turbulent flames using micro-PIV [19]. They reported highly curved upstream propagating fronts, which featured complex dynamics, and observed regions of backflow and static pressure gain in front of the flashbacking flame [19]. In a separate work, the same group of authors [20], investigated the flashback of an open flame into the upstream duct. They considered two cases, in which the flame was initially completely out of the upstream duct or, it was situated slightly inside the duct [20]. It was found that the critical velocity gradient in these two cases were substantially different [20]. These distinctions were attributed to a number of effects including differences in the pressure fields near the reactive region and details of the duct configuration [20]. In all these investigations, the pressure changes in the reactive region were of order of tens of Pascal. Nonetheless, these works [18-20] clearly demonstrated the significant influences of the pressure field on the flame flashback.

Boundary layer flashback of turbulent hydrogen flames has been, recently, subject to DNS studies by Gruber et al. [21]. These authors used detailed chemistry and showed that the leading edge of the propagating reactive front is always very close to the wall and includes significant wrinkles. Back flow pockets and small variations in the cold flow pressure were observed in the vicinity of these wrinkles [21]. It was argued that a hydrodynamic stability (of Darrieus-Landau type) is responsible for this behaviour [21]. On the 
basis of their findings, Gruber et al. [21] considered Lewis and von Elbe criterion [15] to be inadequate for the evaluation of flashback in turbulent boundary layers.

Swirling flows are widely used in gas turbines and industrial burners; as a result there has been a particular interest in this flow configuration [22-24]. Flashback in swirling reactive flows is expected to be in close connection with the subject of flame propagation in vortices. It is known that propagation of premixed flames in vortices can be significantly faster than that in non-rotating flows [25, 26]. In his review article, Ishizuka [27] discusses different mechanisms of this enhancement of flame propagation. In particular, Ishizuka [27] highlights the mechanism of "flame back pressure" which involves a boosted flame propagation supported by an aerodynamic force. This mechanism can be, simply, explained as follows. The combustion products, downstream of the flame, feature a lower density compared to the upstream unburned gases. As a result the negative centrifugal forces in the burned region, are weaker in comparison with those in the upstream unburned flow. Hence, the low pressure generated by the rotation of the flow is partially suppressed, which causes a rise in the static pressure across the reactive front $[27,28]$. This is clearly distinctive with the situation encountered in the non-rotating deflagration waves, in which there is always a drop of static pressure across the flame [29]. In these cases, the decrease of pressure is because of the conversion of static pressure to the flow momentum downstream of a non-rotating premixed front. However, in swirling flames flow, the rotation along with the significant change in the gas density across the flame generates an aerodynamic force [27, 28]. Ishizuka quantified this pressure by the following relation [27]

$$
\Delta P=\rho_{u} V_{\theta_{\max }}^{2}\left[1-\left(\frac{\rho_{b}}{\rho_{u}}\right)\right]
$$

In Eq. $1 P, V$ and $\rho$ are respectively pressure $(\mathrm{Pa})$, velocity $(\mathrm{m} / \mathrm{s})$ and density $\left(\mathrm{kg} / \mathrm{m}^{3}\right)$. Further, indices $\mathrm{b}$, $\mathrm{u}$ and $\theta$ refer to the burned, unburned and tangential components. Conversion of this pressure difference to flow velocity can significantly increase the flame displacement velocity and cause a flashback [28]. It is noted from Eq. 1 that, similar to the pressure differences discussed by Eichler and Sattelmayer [18-20], in typical swirling flames back pressure has a small value and is limited to tens of Pascals. It is, further, noted that a number of studies developed correlations between the flame propagation speed in swirling flows and the maximum circumferential velocity and, density ratio across the flame [28, 30-32]. Some of these correlations have been subject to experimental validations [28]. However, these studies are quite limited and modifications of flame speed due to the tangential motion of the flow remain largely unexplored.

Aerodynamic flame stabilisation is frequently employed in gas turbines and industrial combustors. Flame is stabilised by rotation of the reactants and generation of a recirculating bubble through the phenomenon of vortex breakdown [33]. The occurrence of vortex breakdown, results in the development of a region of low or negative velocity (i.e. a recirculation zone) along the centreline of the rotating flow [33]. This can then stabilise the flame. Despite the large number of studies on vortex breakdown, see [33-35] for reviews of literature, a comprehensive explanation of the phenomenon is yet to emerge [33]. It is well known that there are certain flow features with significant influences on the occurrence of vortex breakdown. Amongst them are the existence of an adverse pressure gradient and sudden expansions in the flow. As most swirling flames may include these features, analogies were proposed between vortex breakdown in isothermal flows and enhanced flame propagation in vortex tubes [36]. In these flows, a high pressure point along the axis of rotation brings the fluid to rest which is, subsequently, followed by the formation of a recirculation zone [36]. 
Vortex breakdown in reactive flows has been investigated in a number of works [36-42]. In an experimental and theoretical work Fritz et al. [36] studied the flashback of a flame purely stabilised by vortex breakdown. These authors demonstrated that the swirling flow field breaks down prior to flashback and therefore encourages upstream propagation of the flame [36]. In comparison with the corresponding isothermal flow, the breakdown point in the combusting flow was located further upstream. Fritz et al. [36] observed that this behaviour is heavily dependent on the configuration of the combustor and a small design change could result in flashback prevention. Hence, a reactive flow can foster the occurrence of vortex breakdown in comparison with its non-reactive counterpart. This was explained as the consequence of pressure changes in the upstream flow induced by the flame heat release [36], but it remained a phenomenological argument without experimentally evaluation. This mechanism was then termed Combustion Induced Vortex Breakdown (CIVB) [36]. In CIVB flame flashback, the flame front propagates upstream of a retarded or reversed flow generated by vortex break down [36].

Experimental studies of Blesinger et al. [43] on swirling flames resulted in the identification of another mechanism of flashback. This was regarded as turbulent burning on the vortex axis (TBVA). Blesinger et al. [43] showed that during TBVA the recirculation zone extends upstream into the mixing tube and turbulent quenching limits axial propagation of the flame The vorticity transport equation applied to a swirling flame was later solved by Kiesewetter et al. [39]. Their analysis showed that the baroclinic torque contributes most significantly with the generation of negative axial velocity [39]. The validity of this analysis was demonstrated by predicting the stability limits of the experiments of Fritz et al. [36]. Further experimental validation was provided by Konle et al. [38] by implementing highspeed PIV and LIF techniques. Investigation of CIVB processes was, then, extended to the interplays between turbulence and chemistry [40, 42]. It should be also noted that there exist some numerical evidence indicated that flame stretch and heat release and also reorientation of vorticity filament could be of significance in CIVB [41, 44]. Later, the interactions between the CIVB and boundary layer flashback, as two major flashback mechanisms were experimentally studied [45]. It was shown that at low equivalence ratios, addition of a low swirl could strengthen the axial flow in the wall region and improve the resistance of the flow against flashback [45]. At higher equivalence ratios, however, the flashback susceptibility increases significantly as the mechanism of combustion induced vortex breakdown is activated. It was observed that once flame penetrates into the upstream tube, bending of the streamlines towards the wall initiates the boundary layer flashback [45]. This then dominates the flame upstream propagation [45]. More recently, the understanding gained from the above studies on boundary layer, vortex break down and core flow flame flashback was used to develop a flashback resistant hydrogen burner [46].

Flame flashback has been also investigated in more complex configurations which include a central bluff-body in a swirling flow [44, 47, 48, 51]. Initial studies of Nauert et al. [47] showed that there exists a critical swirl number above that the flame is destabilised. Any further increase in swirl number can, then, result in flame flashback [47]. Detailed experimental investigation of Heeger et al. [48] demonstrated that the leading front of the flashbacking flame propagates close to the surface of the bluff-body. Hegeer et al. [48] showed that upstream propagation of the flame can be associated with a leading cold flow spot with negative axial velocity. They hypothesised that the flame propagation in the upstream tube generates an adverse pressure gradient, which subsequently causes boundary layer separation on the surface of the bluff-body [48]. This facilitates the upstream propagation of the reactive front and leads to flame flashback. Heeger et al. [48], however, did not conduct any theoretical or experimental analysis on their hypothesis. Later, De and 
Acharya $[49,50]$ conducted large eddy simulations on the flashback of hydrogen enriched premixed flames in a configuration mostly similar to that of Heeger et al. [48]. In keeping with the measurement of Heeger et al. [48], the simulations of De and Acharya [49, 50] revealed the negative velocity spot ahead of the upstream propagating front. Importantly, De and Acharya [49] argued that a positive pressure difference is responsible for the formation of a recirculation bubble ahead of the flashbacking flame. This is induced by the combined effects of centrifugal forces and density jumps across the flame [49]. This argument was, essentially, the same as that put forward by Heeger et al. [48] as a potential deriving mechanism of the flame flashback. Most recently, Karimi et al. [52] elaborated on this idea through the extension of Ishizuka's back pressure theory [27] to a configuration similar to that used in the experiment of Heeger at al. [48]. Karimi et al. showed that their calculated pressure difference across the flame was similar to the experimentally measured values [52]. They argued that this adverse pressure gradient could contribute with the observed flame flashback [48] through separating the boundary layer, on the surface of the bluff-body [52]. Importantly, other recent experimental and theoretical [18-20, 44] and numerical [49] investigations have also highlighted the significance of adverse pressure gradient upon flashback in boundary layers. Further, pressure changes can contribute with the baroclinic torque, which is known as a key element in CIVB [39, 49, 50]. It follows that the detection of all mechanisms of generating adverse pressure gradient is central to the understanding of flame flashback in configurations involving swirling flows and a central bluff-body.

Theoretical analyses of upstream propagation of swirling flames, so far, have solely considered the axial motion of the flame and totally ignored the circumferential propagation [27, 52]. Images of flame during flashback, however, indicate the existence of a strong circumferential motion of the flame around the bluff-body [48, 51]. In principle, the angular motion of the flame and the density difference across the flame can generate a positive pressure gradient in the circumferential direction. However, currently there is no systematic analysis of this mechanism. This paper, therefore, aims at addressing this issue through a theoretical investigation. The outcomes are, subsequently, compared against the experimental measurements.

It should be emphasised here that, as the preceding review of literature indicates, our current understating of flame flashback is still far from being complete. Flame flashback is a complex phenomenon which includes significant physicochemical and fluid mechanical aspects. In addition to these, the phenomenon is highly transient and features complicated dynamics. This makes numerical studies of flame flashback very challenging. High speed diagnostics can provide useful information on flashbacking flames. Nonetheless, due to the complexity of the problem interpretation of the experimental results is usually difficult. These limitations signify the role of theoretical analyses in understanding of flame flashback. Expectedly, these studies require considerable simplifications and therefore can only analyse a part of the problem. The purpose of the theoretical investigations is not to provide predictive models of flashback and they should not be deemed as a substitution for high fidelity numerical simulations. They, rather, tend to develop a sound understanding of certain physical mechanisms active in the process of flame flashback.

\section{Experimental Analysis}

Upstream flame propagation was experimentally investigated in an unconfined, swirling, lean, premixed, bluff-body stabilised burner. The flame flashback in this setup occurs 
through a combined circumferential and axial motion against the cold flow. Schneider et al. [51] provide comprehensive explanation of the employed setup. Further modifications to enable detailed observation of the upstream flame propagation were made by Nauert et al. [47]. Hence, in here only the essential features are briefly reviewed. Air and fuel (pure methane) are mixed inside the plenum before entering the radial swirler and the exit nozzle. The movable block design allowed variations in the geometric swirl number between 0 and 2 through rotating the movable block by a stepper motor. To provide optical access the exit nozzle was made of quartz. For the test cases presented in here, the Reynolds number, on the basis of hydraulic diameter of the nozzle, was 10,000 and the equivalence ratio was set to 0.833 . The onset of flashback was induced by increasing the swirl number as explained by Nauert et al. [47]. Flame flashback occurred when the value of theoretical swirl number approached one [47]. Piezo-resistive pressure transducers (Omegadyne Inc. PX 409) were used to measure the pressure on the surface of the bluff-body. Remote location of the sensors from the hot gas region was a prerequisite to prevent any damage. To accommodate the pressure transducers and protect them from the hot flow, the bluff-body design was modified. A number of pressure measurement ports with the diameter of $1 \mathrm{~mm}$ were built inside the bluff-body. These were connected to pressure sensors by internal channels with equal length of $300 \mathrm{~mm}$.

The total pressure measurement period was $1 \mathrm{~s}$ and started shortly before flashback. High speed imaging was performed simultaneously and showed that flame upstream propagation occurs in a small fraction of a second. This period was, therefore, enough to capture the entire flashback event. The analogue signal produced by the microphone was digitised and recorded by an oscilloscope (Tektronix) at $2 \mathrm{MHz}$. Information about the global flame position and structure were acquired by flame chemiluminescence (CL) imaging of electronically excited methylidyne, $\mathrm{CH}^{*}$, with a high speed CMOS camera (HSS5) at $5 \mathrm{kHz}$ frame rate. The imaging region was $60 \mathrm{~mm} \times 60 \mathrm{~mm}$ to cover the whole transparent nozzle. These measurements were taken with simultaneous and time correlated acquisition of the pressure transducer signals.

Detailed analyses of the cold and reactive velocity fields of the configuration under investigation can be found in the Refs. [47, 48, 51] and are not repeated here. Further, Ref. [49] provides the streamline patterns of a flashbacking flame in a configuration quite similar to the current setup. Figure 2 shows the results of synchronised CL and single-port pressure measurements during a flame flashback event. It is worth noting that the pressure traces measured at other pressure ports on the bluff body (see Fig. 1) look quite similar to that shown in Fig. 2. Image (a) in this sequence is recorded prior to flashback. In this figure the precessing flame is still downstream of the location where the pressure is monitored. In image (f) the flame already flashed back and has entered the upstream sections of the burner. The pressure fluctuations are large and due to a thermoacoustic combustion instability of the resonance frequency of the entire nozzle assembly [51, 52]. It was shown, previously, that this instability is a consequence of upstream flame propagation and therefore does not affect the process of flashback [52]. Image sequence (b) to (e) corresponds to the duration that the reactive front passes across the pressure sensing location (every tenth frame of chemiluminescence imaging shown). These images were selected out of hundreds of images taken in this period. It is clear from the flame images in Fig. 2 that propagation of the front is associated with an increase in the local static pressure. This is in keeping with the previous experimental observations [52]. These images also highlight the significance of the tangential propagation of the reactive front. Although a vertical upstream motion of the flame does exist, Fig. 2b-e clearly show a strong circumferential motion of the flame during the flashback event. 


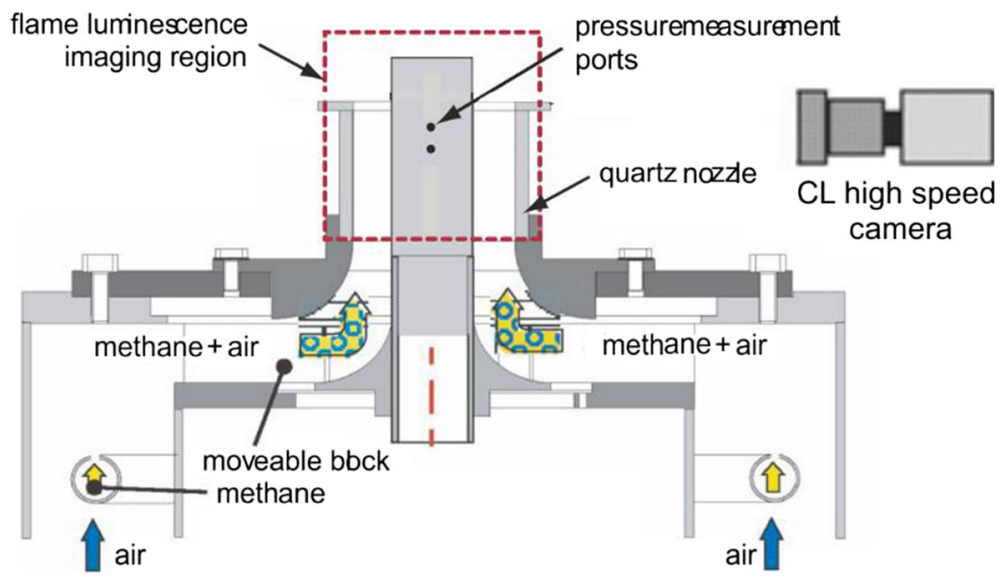

Fig. 1 Schematic of the premixed swirl burner

\section{Theoretical Analysis}

Figure 3 schematically shows the theoretical problem under investigation. The flow system includes a tube with a central bluff-body fully filled by a Rankin vortex, while the axial velocity is set to zero. The analysis assumes that there exists a laminar flame propagating against the mean rotating flow. A control volume approach is taken and the governing equations are solved in their integral form. Clearly, this analysis is limited to the durations shorter than that required for a complete rotation of the flame around the bluff-body. The current study aims at understanding the mechanisms responsible for developing an adverse pressure gradient across the flame in the setup shown in Fig. 3. It is noted that this is a rather idealised configuration and in reality flame propagation will be more involved. However, the current setting provides a proper basis for the analysis of the pressure differences across a rotating flame in isolation from all other complicating effects. The theoretical results are, therefore, not meant to be quantitatively accurate and only indicate the overall qualitative trends.
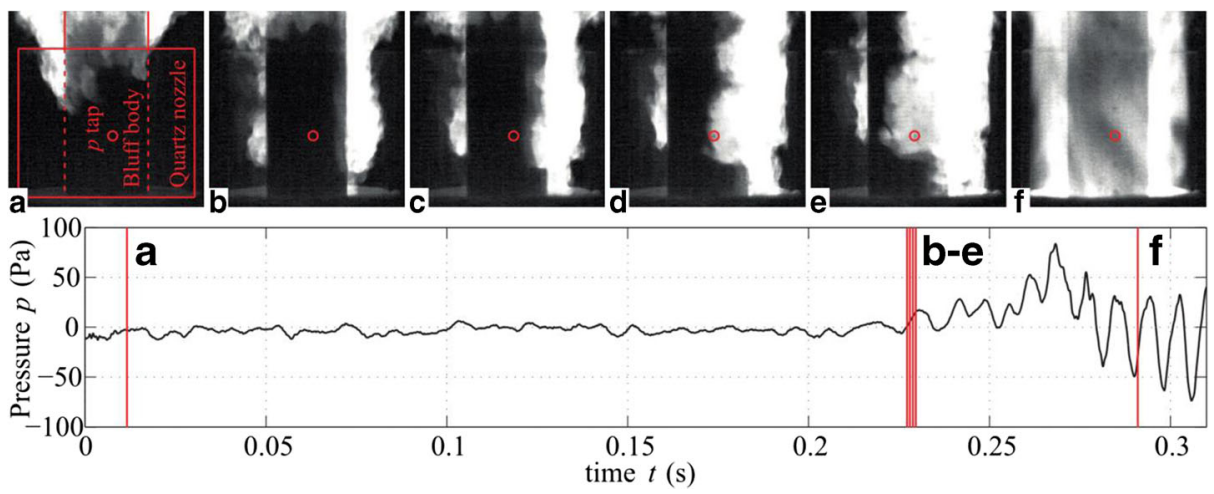

Fig. 2 Pressure trace prior to a, during b-e, and after flashback $\mathbf{f}$. The location of pressure recording is marked by the red circle (upper row) 


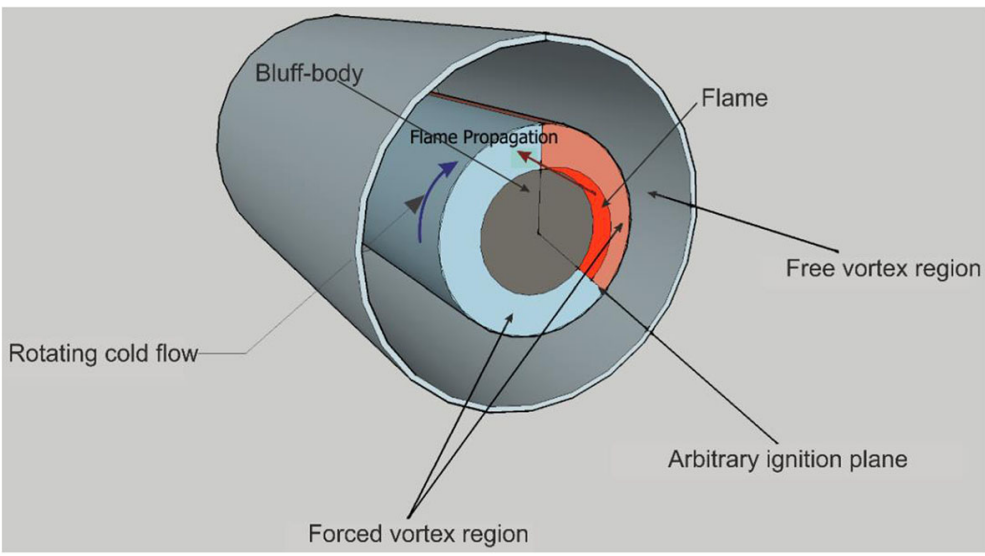

Fig. 3 Schematic view of the problem

\subsection{Governing equations and assumptions}

The following assumptions are made throughout the proceeding analysis.

- Flow is inviscid and, the circumferential velocities in the burned and unburned regions are uniform and follow a classical Rankin vortex model.

- Axial flow velocity is globally zero and the gravitational effects are ignored.

- Flame is laminar and premixed and has zero thickness.

- The control volume containing the flame (see Fig. 4) is rotating with the flame at a constant rotational speed (steady flame rotation).

- Burning only occurs in the forced vortex region of the flow, and the entire volume between the forced vortex core and the walls of the cylinder is filled by the free vortex.

- The flame is completing its first rotation around the bluff-body.

A sector of the flow under investigation, including the burned and unburned regions, has been shown in Fig. 4. The employed symbols have been defined in the nomenclature.

The conservation of mass and radial momentum for a non-deformable control volume rotating at constant angular velocity are respectively written as

$$
\iint \rho\left(V_{r} \cdot \hat{n}\right) d A=0
$$

Fig. 4 The control volume including the flame (red), forced vortex (grey) and free vortex (white)

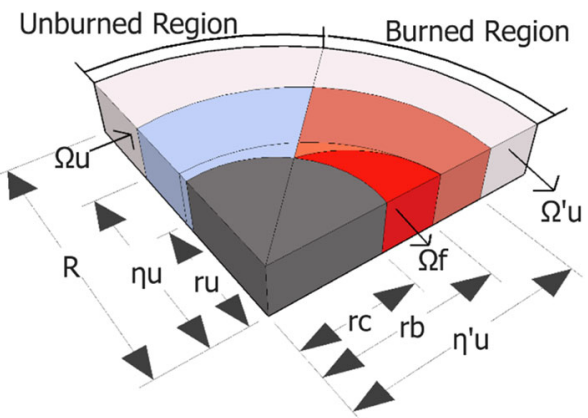


and

$$
\frac{d P}{d r}=\frac{\rho V_{\theta}^{2}}{r}+2 \Omega_{f}^{2} r
$$

Further, in this rotating coordinate Bernoulli equation can be written for the streamline located on the internal wall of the cylinder $(r=R)$. That is

$$
P_{u}(R)+\left.\frac{\rho_{u} V_{\theta_{R}}^{2}}{2}\right|_{u}=P_{b}(R)+\left.\frac{\rho_{u} V_{\theta_{R}}^{2}}{2}\right|_{b},
$$

where $V_{\theta_{R}}$ is defined as

$$
V_{\theta_{R}}=V_{\theta}-\Omega r,
$$

and subscripts ' $u$ ' and ' $b$ ' are the unburned and burned regions respectively and $R$ is the internal radius of the wall of the cylinder. It should be noted that, as Figs. 3 and 4 show, the internal wall is always in contact with the cold gas.

The tangential velocity, $V_{\theta}$, is assumed to follow the form of a Rankine vortex with an axial velocity of zero. Thus,

$$
\begin{gathered}
V_{z}=0 \text { for all values of } \mathrm{r}, \\
V_{\theta}=\left\{\begin{array}{l}
\Omega r \text { for }(0 \leq r \leq \eta) \\
\frac{\Omega \eta^{2}}{4 r} \text { for }(\eta \leq r \leq R)^{\prime}
\end{array}\right.
\end{gathered}
$$

A non-dimensional cold flow angular velocity is, further, defined on the basis of laminar flame velocity of the fuel and radius of the duct. This reads

$$
\omega^{*}=\frac{\Omega_{u}}{\frac{S_{u}}{R}}
$$

\subsection{Model development}

The current analysis builds upon Ishizuka's original theory of flame back pressure [27]. This theory is extended to the configuration shown Fig. 3 in which flame propagates solely circumferentially. The following vortex regions are first defined:

- burned vortex region $\left\{r_{c}<r<r_{b}\right\}$,

- unburned forced vortex region $\left\{r_{b}<r \leq \eta_{u}^{\prime}\right\}$,

- unburned free vortex region $\left\{\eta_{u}^{\prime}<r \leq R\right\}$.

These confined vortex regions are shown in Fig. 3, and in more details in Fig. 4. The analysis starts by applying the conservation of mass (2) to the three separate control volumes defined as the burned, unburned forced and unburned free vortex regions. This yields the followings in the burned forced vortex

$$
\left(\Omega_{u}-\Omega_{f}\right)\left(r_{u}^{2}-r_{c}^{2}\right)=\frac{\rho_{b}}{\rho_{u}}\left(\Omega_{b}-\Omega_{f}\right)\left(r_{b}^{2}-r_{c}^{2}\right),
$$

within the unburned forced vortex:

$$
\left(\Omega_{u}-\Omega_{f}\right)\left(\eta_{u}^{2}-r_{u}^{2}\right)=\left(\Omega_{u}^{\prime}-\Omega_{f}\right)\left(\eta_{u}^{\prime 2}-r_{b}^{2}\right),
$$

and in the unburned free vortex,

$$
\frac{\Omega_{u} \eta_{u}^{2}}{4} \ln \left(\frac{R}{\eta_{u}}\right)-\frac{\Omega_{f}}{2}\left(R^{2}-\eta_{u}^{2}\right)=\frac{\Omega_{u}^{\prime} \eta_{u}^{\prime 2}}{4} \ln \left(\frac{R}{\eta_{u}^{\prime}}\right)-\frac{\Omega_{f}}{2}\left(R^{2}-\eta_{u}^{\prime 2}\right) .
$$


The rotational velocities in these regions are then determined through using the Rankine vortex model (6 and 7). For the regions upstream of the flame,

$$
V_{u}=\left\{\begin{array}{l}
\Omega r \text { for }\left(r_{c} \leq r \leq \eta_{u}\right) \\
\frac{\Omega \eta_{u}^{2}}{4 r} \text { for }\left(\eta_{u} \leq r \leq R\right)^{\prime}
\end{array}\right.
$$

and for the regions of the flow downstream of the flame

$$
V_{b}=\left\{\begin{array}{l}
\Omega_{b} r \text { for }\left(r_{c} \leq r \leq r_{b}\right) \\
\Omega_{u}^{\prime} r \text { for }\left(r_{b} \leq r \leq \eta_{u}^{\prime}\right) \\
\frac{\Omega_{u}^{\prime} \eta_{u}^{\prime 2}}{4 r} \text { for }\left(\eta_{u}^{\prime} \leq r \leq R\right)
\end{array} .\right.
$$

The linear and angular flame speeds are related as follows

$$
V_{f}=\Omega_{f} r \text { for all } \mathrm{r} \text {. }
$$

Applying Eq. 3 to the burned and unburned regions, results in an expression for the conservation of radial momentum in the rotating coordinate. Carrying out the integrations yields the pressure differences upstream and downstream of the flame in the rotating frame. After some algebra it can be shown that for the unburned region

$$
P_{u}(R)-P_{u}\left(r_{c}\right)=\rho_{u} \Omega_{u}^{2} \frac{\left(\eta_{u}^{2}-r_{c}^{2}\right)}{2}-\rho_{u} \frac{\Omega_{u}^{2} \eta_{u}^{4}}{32}\left(\frac{1}{R^{2}}-\frac{1}{\eta_{u}^{2}}\right)+\rho_{u} \Omega_{f}^{2}\left(R^{2}-r_{c}^{2}\right),
$$

and in the burned region

$$
\begin{aligned}
P_{b}(R)-P_{b}\left(r_{c}\right)= & \frac{\rho_{u} \Omega_{u}^{\prime 2} \eta_{u}^{\prime 4}}{32}\left(\frac{1}{R^{2}}-\frac{1}{\eta_{u}^{\prime 2}}\right)+\frac{\rho_{b} \Omega_{b}^{2}\left(r_{b}^{2}-r_{c}^{2}\right)}{2}+\frac{\rho_{u} \Omega_{u}^{\prime 2}\left(\eta_{u}^{\prime 2}-r_{b}^{2}\right)}{2} \\
& +\rho_{b} \Omega_{f}^{2}\left(r_{b}^{2}-r_{c}^{2}\right)+\rho_{u} \Omega_{f}^{2}\left(R^{2}-r_{b}^{2}\right) .
\end{aligned}
$$

Further, Eq. 4, the Bernoulli equation for a streamline at the wall of the cylinder $(r=R)$ can be written as

$$
P_{u}(R)+\frac{\rho_{u} \Omega_{u}^{2} \eta_{u}^{4}}{32 R^{2}}-\frac{\rho_{u} \Omega_{u} \Omega_{f} \eta_{u}^{2}}{4}=P_{b}(R)+\frac{\rho_{u} \Omega_{u}^{\prime 2} \eta_{u}^{\prime 2}}{32 R^{2}}-\frac{\rho_{u} \Omega_{u}^{\prime} \Omega_{f} \eta_{u}^{\prime 2}}{4} .
$$

To make the analysis more concise, the following parameters are defined. The expansion ratio is

$$
\epsilon_{r}=\frac{r_{b}}{r_{u}}
$$

and the burning ratio is defined as,

$$
k=\frac{r_{u}}{\eta_{u}} .
$$

Ratio of the bluff body radius to the unburned forced vortex radius upstream is termed $K^{\prime}$. That is

$$
K^{\prime}=\frac{r_{c}}{\eta_{u}}
$$

and the ratio of the unburned forced vortex radius upstream to the radius of the outer wall of the cylinder, is regarded as $K$ :

$$
k=\frac{\eta_{u}}{R} \text {. }
$$

Expansion ratio corrected for the presence of bluff-body is written as

$$
\epsilon_{r}^{\prime \prime}=\frac{\left(\epsilon_{r} k\right)^{2}-K^{\prime 2}}{k^{2}-K^{\prime}},
$$


and the density ratio is defined as,

$$
\delta=\frac{P_{u}}{P_{b}}
$$

Downstream angular velocity of the cold flow can be related to that of upstream through applying the conservation of the radial momentum. Thus,

$$
\Omega_{u}^{\prime}=\frac{\eta_{u}^{2}}{\eta^{\prime 2}} \Omega_{u}
$$

As this equation contains the unknown parameter of $\eta_{u}^{\prime}$, the following ratio is introduced. The ratio for the expansion of the upstream to downstream forced vortex radii, $\epsilon_{r}^{\prime}$, is defined as

$$
\epsilon_{r}^{\prime}=\frac{\eta_{u}^{\prime}}{\eta_{u}}
$$

Substituting Eq. 25 into Eq. 24 leads to an expression for the angular velocity of the unburned gas downstream of the flame,

$$
\Omega_{u}^{\prime}=\frac{1}{\epsilon_{r}^{\prime 2}} \Omega_{u} .
$$

To solve for the flame angular velocity, $\Omega_{f}$, Eqs. 25 and 26 are substituted into Eq. 10. This yields,

$$
\Omega_{f}=\Omega_{u} \frac{\left(\frac{r_{b}^{2}}{\epsilon^{\prime 2}} r_{u}^{2}\right)}{\left(\eta_{u}^{2}-\eta_{u}^{2}-\eta_{u}^{2} \epsilon_{r}^{\prime 2}+r_{b}^{2}\right)} .
$$

Substituting the non-dimensional ratios defined in Eqs. 18 and 19 into Eq. 10 results in the following expression for the rotational speed of the flame,

$$
\Omega_{f}=\Omega_{u} k^{2} \frac{\left[\left(\frac{\epsilon_{r}}{\epsilon_{r}^{\prime}}\right)^{2}-1\right]}{\left[1-k^{2}-\epsilon^{\prime 2}+\left(\epsilon_{r} k\right)^{2}\right]} .
$$

To find an expression for the burning rotational speed, $\Omega_{b}$, Eqs. 22, 23 and 28 are substituted into Eq. 9 resulting in

$$
\Omega_{b}=\Omega_{u}\left[\left(1-k^{2} \frac{\left[\left(\frac{\epsilon_{r}}{\epsilon_{r}^{\prime}}\right)^{2}-1\right]}{\left[1-k^{2}-\epsilon_{r}^{\prime 2}+\left(\epsilon_{r} k\right)^{2}\right]}\right) \frac{\delta}{\epsilon_{r}^{\prime \prime}}+k^{2} \frac{\left[\left(\frac{\epsilon_{r}}{\epsilon_{r}^{\prime}}\right)^{2}-1\right]}{\left[1-k^{2}-\epsilon_{r}^{\prime 2}+\left(\epsilon_{r} k\right)^{2}\right]}\right] .
$$

We now refer back to Eq. 25 which includes two unknowns. An approach to solving this is to find the ratio $\epsilon_{r}^{\prime}$ first and then use that to determine $\eta_{u}^{\prime}$. Substituting Eqs. 25 and 28 into Eq. 11 yields,

$$
\operatorname{In}\left(\epsilon_{r}^{\prime}\right)\left[1-k^{2}-\epsilon_{r}^{\prime 2}+\left(\epsilon_{r} k\right)^{2}\right]=2 k^{2}\left[\epsilon_{r}^{2}-\left(\frac{\epsilon_{r}}{\epsilon_{r}^{\prime}}\right)^{2}-\epsilon_{r}^{\prime 2}+1\right] .
$$

The left hand side of Eq. 30 features an issue for solving for $\epsilon_{r}^{\prime}$ as a function of $k$ and $\epsilon_{r}$. This is due to the existence of logarithmic function in this equation, which makes the analytical solution difficult. To resolve this issue a Taylor series expansion of $\ln \epsilon_{r}^{\prime}$ is employed. This provides an estimated value of $\epsilon_{r}^{\prime}$ which in turn can be used in Eq. 25 to find $\eta_{u}^{\prime}$.

Appendix A provides the details of the polynomial coefficients from the first to fourth order expansion of Eq. 30. Assuming that $\epsilon_{r}^{\prime}$ takes values close but greater than one the first few terms on this series can be considered a good approximation of $\ln \epsilon_{r}^{\prime}$. 
Substituting Eqs. 15 and 16 into Eq. 17 results in the following relation

$$
\begin{array}{r}
P_{u}\left(r_{c}\right)-P_{b}\left(r_{c}\right)=\left[\frac{\rho_{b} \Omega_{b}^{2}\left(r_{b}^{2}-r_{c}^{2}\right)}{2}-\frac{\rho_{u} \Omega_{u}^{\prime 2} \eta_{u}^{\prime 4}}{32}\left(\frac{1}{R^{2}}-\frac{1}{\eta_{u}^{\prime 2}}\right)+\frac{\rho_{u} \Omega_{u}^{\prime 2}\left(\eta_{u}^{\prime 2}-r_{b}^{2}\right)}{2}(31) \Omega_{f}^{\prime 2} \Omega_{f}^{2}\left(r_{b}^{2}-r_{c}^{2}\right)+\rho_{u} \Omega_{f}^{2}\left(R^{2}-r_{c}^{2}\right)+\frac{\rho_{u} \Omega_{u}^{\prime 2} \eta_{u}^{\prime}}{32 R^{2}}-\frac{\rho_{u} \Omega_{u}^{\prime} \Omega_{f} \eta_{u}^{\prime 2}}{4}\right]\left[\frac{\rho_{u} \Omega_{u}^{2}\left(\eta_{u}^{2}-r_{c}^{2}\right)}{2}\right. \\
+\rho_{u} \Omega_{u}^{2} \eta_{u}^{4} \\
\left.\frac{\rho^{2}}{32}\left(\frac{1}{R_{2}}-\frac{1}{\eta_{u}^{2}}\right)+\rho_{u} \Omega_{f}^{2}\left(R^{2}-r_{c}^{2}\right)+\frac{\rho_{u} \Omega_{u}^{2} \eta_{u}^{4}}{32 R^{2}}-\frac{\rho_{u} \Omega_{u} \Omega_{f} \eta_{u}^{2}}{4}\right] .
\end{array}
$$

Application of Eqs. 18-29 turns Eq. 32 into its non-dimensional form. That is

$$
\begin{aligned}
\frac{(\Delta P)_{r_{c}}}{\rho_{u} \Omega_{u}^{2} \eta_{u}^{2}}=\left[\frac { ( ( \epsilon _ { r } k ) ^ { 2 } - K ^ { \prime 2 } ) } { 2 \delta } \left[\frac{\delta^{2}}{\epsilon_{r}^{\prime \prime 2}}-k^{2} \frac{2 \delta^{2}}{\epsilon_{r}^{\prime \prime 2}} \frac{\alpha}{\beta}+k^{2} \frac{2 \delta}{\epsilon_{r}^{\prime \prime}} \frac{\alpha}{\beta}-k^{4} \frac{2 \delta}{\epsilon_{r}^{\prime \prime 2}}\left(\frac{\alpha}{\beta}\right)^{2}\right.\right. \\
\left.+k^{2} \frac{\delta^{2}}{\epsilon_{r}^{\prime \prime 2}}\left(\frac{\alpha}{\beta}\right)^{2}+k^{4}\left(\frac{\alpha}{\beta}\right)^{2}\right]+\left\{\left[\frac{1}{32}\left(\epsilon_{r}^{\prime 2}-1\right)\right]+\frac{1}{2}\left[\frac{\left(\epsilon_{r}^{\prime 2}-\left(\epsilon_{r} k\right)^{2}\right)}{\epsilon_{r}^{\prime 4}}-\left(1-K^{\prime 2}\right)\right]\right\} \\
\left.+\frac{\left(\epsilon_{r} k\right)^{2}-K^{\prime 2}}{2 \delta} k^{4}\left(\frac{\alpha}{\beta}\right)^{2}-\left(\left(\epsilon_{r} k\right)^{2}-K^{\prime 2}\right) k^{4}\left(\frac{\alpha}{\beta}\right)^{2}\right] .
\end{aligned}
$$

In which $(\Delta P)_{r_{c}}$ is the static pressure difference between the burned and unburned gases on the surface of the bluff-body. Further,

$$
\alpha=\left(\frac{\epsilon_{r}}{\epsilon_{r}^{\prime}}\right)^{2}-1
$$

and

$$
\beta=1-k^{2}-\epsilon_{r}^{\prime 2}+\left(\epsilon_{r} k\right)^{2} .
$$

Finally, non-dimensionalising angular velocity and vortex thickness turns Eq. 33 to

$$
\begin{aligned}
\frac{(\Delta P)_{r_{c}}}{\rho_{u} \omega^{* 2} S_{u}^{2} K^{2}}= & {\left[\frac { ( ( \epsilon _ { r } k ) ^ { 2 } - K ^ { \prime 2 } ) } { 2 \delta } \left[\frac{\delta^{2}}{\epsilon_{r}^{\prime \prime 2}}-k^{2} \frac{2 \delta^{2}}{\epsilon_{r}^{\prime \prime 2}} \frac{\alpha}{\beta}+k^{2} \frac{2 \delta}{\epsilon_{r}^{\prime \prime}} \frac{\alpha}{\beta}-k^{4} \frac{2 \delta}{\epsilon_{r}^{\prime \prime 2}}\left(\frac{\alpha}{\beta}\right)^{2}\right.\right.} \\
& \left.+k^{4} \frac{\delta^{2}}{\epsilon^{\prime \prime 2} r}\left(\frac{\alpha}{\beta}\right)^{2}+k^{4}\left(\frac{\alpha}{\beta}\right)^{2}\right] \\
& +\left\{\left[\frac{1}{32}\left(\epsilon_{r}^{\prime 2}-1\right)\right]+\frac{1}{2}\left[\frac{\left(\epsilon_{r}^{\prime 2}-\left(\epsilon_{r} k\right)^{2}\right)}{\epsilon_{r}^{\prime 4}}-\left(1-K^{\prime 2}\right)\right]\right\} \\
& \left.+\frac{\left(\left(\epsilon_{r} k\right)^{2}-K^{\prime 2}\right)}{2 \delta} k^{4}\left(\frac{\alpha}{\beta}\right)^{2}-\left(\left(\epsilon_{r} k\right)^{2}-K^{\prime 2}\right) k^{4}\left(\frac{\alpha}{\beta}\right)^{2}\right],
\end{aligned}
$$


Table 1 Values of the parameters used in the theoretical analysis

\begin{tabular}{llllll}
\hline$\delta=\frac{\rho_{u}}{\rho_{b}}$ & $\epsilon_{r}=\frac{r_{b}}{r_{u}}$ & $k=\frac{r_{u}}{\eta_{u}}$ & $K^{\prime}=\frac{r_{c}}{\eta_{u}}$ & $K=\frac{\eta_{u}}{R}$ & $\omega^{*}$ \\
\hline 7 & 1.5 & 0.6 & 0.5 & 0.2 & 25 \\
\hline
\end{tabular}

where $\omega^{*}$ and $K$ are defined in Eqs. 8 and 21, respectively. The expression for the flame angular velocity $\Omega_{f}$, as shown in Eq. 28 , can be expressed in terms of the non-dimensional angular velocity by using Eq. 8. This reveals the following expression,

$$
\Omega_{f}=\left(\frac{\omega^{*} S_{u}}{R}\right) k^{2} \frac{\left[\left(\frac{\epsilon_{r}}{\epsilon_{r}^{\prime}}\right)^{2}-1\right]}{\left[1-k^{2}-\epsilon_{r}^{\prime 2}+\left(\epsilon_{r} k\right)^{2}\right]} .
$$

\section{Theoretical Results and Discussion}

The model developed in Section 3 expresses the pressure difference across the flame and also flame angular velocity as a function of the flow and geometrical parameters. The effects of these parameters along with the physical behaviour of the developed solutions are discussed in this section. Unless specified otherwise, the numerical values of the parameters used in the current analysis are those listed in Table 1 . These values have been selected to represent a typical flow configuration. The influences of the variations of these parameters will be discussed throughout this section.

Considering the values in Table 1 and solving Eq. 31 through approximating the logarithmic term with the first four terms of the series expansion, yields $\epsilon_{r}^{\prime}=\frac{\eta_{u}^{\prime}}{\eta_{u}} \approx 1.639$. Further, the current analysis uses the non-dimensional form of the pressure difference across the flame, the laminar flame speed and the flame angular velocity. These are defined in Table 2. In Figs. 5, 6, 7, 8, 9, 10 and $11 P_{u}$ takes the value of the atmospheric pressure and the laminar flame velocity changes from zero to its typical value for the gaseous hydrocarbons and air mixtures $\left(0.4 m s^{-1}\right)$.

Figure 5 shows the effects of the external tube radius and laminar flame speed upon the pressure difference across the flame. The former has been expressed as the ratio of the cold vortex thickness $\left(\eta_{u}\right)$ and the tube radius (R), collectively represented by $K=\frac{\eta_{u}}{R}$, and the latter takes the non-dimensional form shown in Table 2. It is clear in Fig. 5 that the pressure difference approaches zero in the limit of zero laminar flame speed. This is to be expected as the pressure difference is essentially due to the changes in the centrifugal forces on the two sides of the flame, induced by combustion [27]. Zero laminar flame speed implies no combustion and therefore the pressure difference should disappear in this cold flow limit. For finite values of laminar flame speed and for all the considered values of the ratio of vortex thickness and tube radius, the non-dimensional pressure difference has a negative value.

Table 2 Non-dimensional groups

\begin{tabular}{lll}
\hline $\begin{array}{l}\text { Non-dimensional } \\
\text { pressure difference }\end{array}$ & $\begin{array}{l}\text { Non-dimensional } \\
\text { laminar flame speed }\end{array}$ & $\begin{array}{l}\text { Non-dimensional flame } \\
\text { angular velocity }\end{array}$ \\
\hline$\left(\frac{1}{P_{u}}\right) \Delta P=\left(\frac{1}{P_{u}}\right)\left(P_{u}-P_{b}\right)$ & $\left(\frac{\rho_{u}}{P_{u}}\right) S_{u}^{2}$ & $\Omega_{f}\left(\frac{R}{S_{u}}\right)$ \\
\hline
\end{tabular}




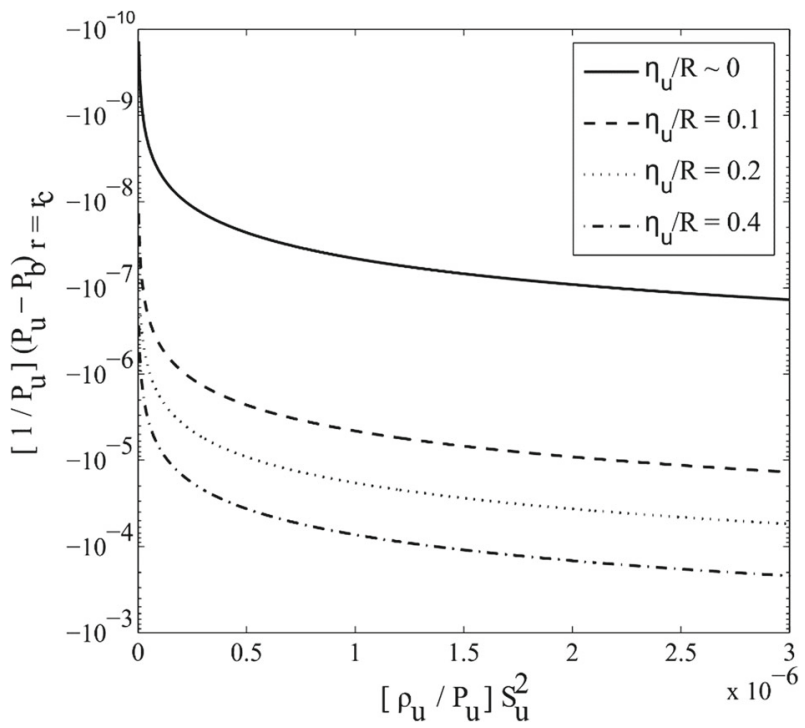

Fig. 5 Effect of the radius of the external tube on the pressure difference across the flame for varying dimensionless flame speed

This indicates that the static pressure in the burner region is greater than that of the cold flow. Hence, the circumferential propagation of the flame is associated with the development of an adverse pressure gradient. This is clearly different to that observed in the axial propagation of a non-rotating premixed front, in which the static pressure drops in the burned region

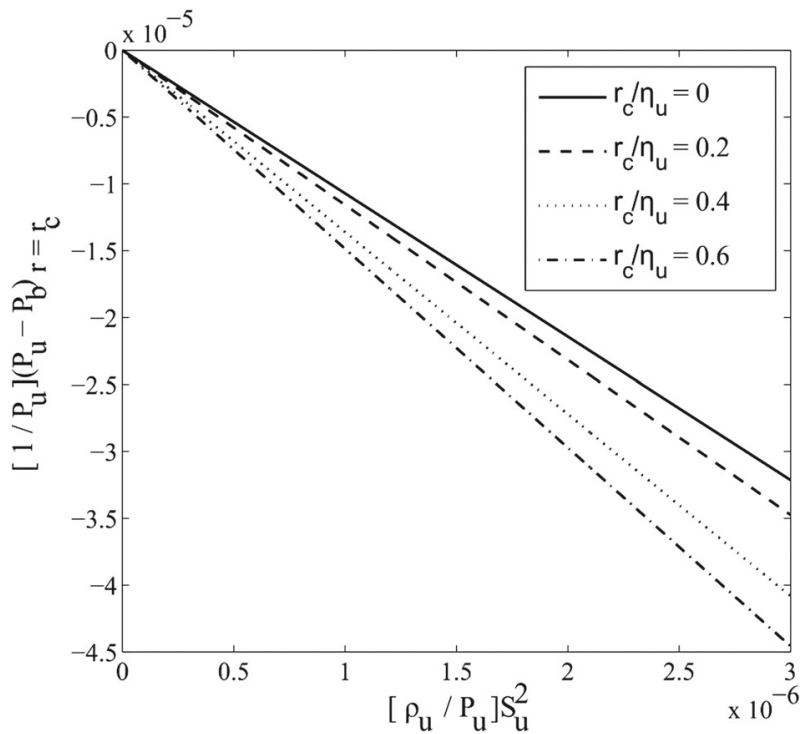

Fig. 6 Effect of the bluffbody radius on the pressure difference across the flame for varying dimensionless flame speed 


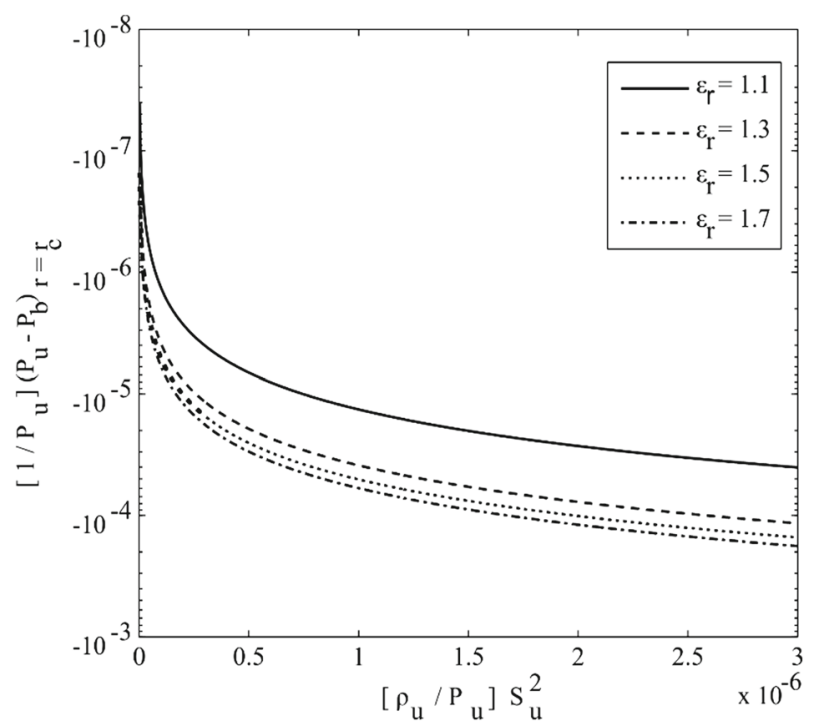

Fig. 7 Effect of variations in the expansion ratio on the pressure difference across the flame for varying dimensionless flame speed

$[15,29]$. It is noted that for all values of $\frac{\eta_{u}}{R}$, increasing the laminar flame speed signifies the pressure difference across the flame. Once again, this is consistent with the physical intuition. Here, more reactive flames, propagating at higher speed, cause stronger changes in the tangential momentum thus induce higher pressure difference across the flame. The same qualitative behaviour has been reported previously in the axially propagating, swirling flames [52]. Further, it is observed, in Fig. 5, that increasing $\frac{\eta_{u}}{R}$ (or decreasing the tube radius) strengthens the pressure difference across the flame. Notably, Fig. 5 demonstrates the strong effect of confinement upon the back pressure mechanism in the present circumferential flashback. For the case with no confinement $\left(\eta_{u} / R \sim 0\right.$, implying large values of $R$ ) the pressure gain remains negligibly small. Nonetheless, by increasing the confinement (i.e. increasing $\eta_{u} / R$ ) the developed pressure difference across the flame rises quickly. This trend is in keeping with that previously observed in the axial flashback of rotating flames [52].

A similar behaviour is observed in Fig. 6, which shows the influence of the bluff-body radius on the pressure difference across the flame for varying dimensionless flame speeds. In this figure, the adverse pressure gradient increases for larger bluff-body radii. This could be interpreted as increasing the flow confinement. As observed in Fig. 5, the more confined system develops a larger pressure difference. Figures 5 and 6 imply that a narrow flow passage between the tube and bluff-body can substantiate the pressure difference across the flame. Nonetheless, as explained in Ref. [52], this is subject to maintaining all other parameters, in Table 1, constant. It should be also stressed that the effect of changes in the external tube radius appears to be much stronger than that due to the variations in bluff-body radius. Further, comparing to the effects of varying the external tube radius, the changes in the bluff-body radius has a more uniform effect on the pressure gain.

Figure 7 illustrates the sensitivity of the developed back pressure upon the expansion ratio, $\epsilon_{r}$. The practical value of this parameter is not precisely known and depends on a number of parameters including the bluff-body radius, flow regime and characteristics and also 


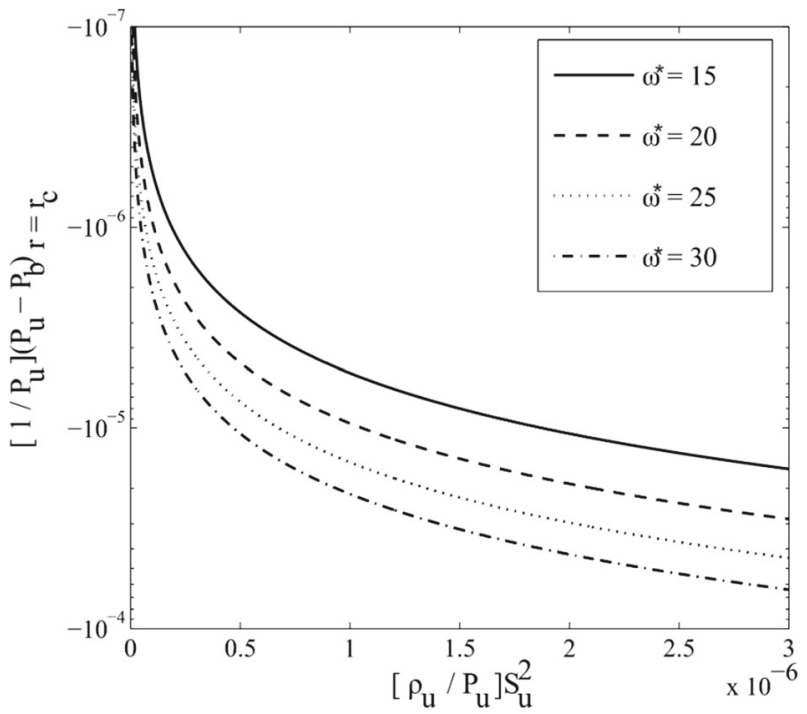

Fig. 8 Effect of altering the upstream angular velocity on the pressure difference across the flame, for varying dimensionless flame speed

the fuel exothermicity. This figure shows that, in general, by decreasing the expansion ratio the pressure difference across the flame drops. The decrease in the pressure gain appears to be non-uniform and intensifies as the expansion ratio approaches one. This behaviour is due to the nonlinear nature of Eq. 35 with respect to $\epsilon_{r}$, and is also expected from the derivation of the model in Section 3.2. It is important to note that, similar to the original work of Ishizuka [27], the current study treats the expansion and density ratios as two independent variables. Decreasing each of these two parameters results in the weakening of the back pressure mechanism and decreases the pressure difference across the flame.

Figure 8 demonstrates the effects of cold flow angular velocity upon the pressure difference as the laminar flame speed varies. Similar to that observed in Fig. 5 the pressure difference vanishes in the limit of zero laminar flame speed. It is also clear from this figure that higher angular velocities of the cold flow result in stronger pressure difference across the flame. Angular velocity of the cold flow correlates directly with the tangential momentum of the rotating cold flow. Hence, for a given density and expansion ratio and laminar flame speed, the faster rotating flow generates a more significant change in the tangential momentum and hence produces a larger pressure difference.

In the model developed in Section 3, only a fraction of the gas in the forced vortex burns. A measure of this fraction is provided by the burning ratio, $k \equiv r_{u} / \eta_{u}$. This represents the ratio of the thickness of a layer of the cold gas which ultimately burns, to the thickness of the forced vortex. Figure 9 shows the calculated pressure difference for a few values of the burning ratio. It is clear in this figure that as the numerical value of $k$ increases the pressure gain decreases significantly (note that by definition $k>K^{\prime}$, as $r_{u}>r_{c}$ ). It is difficult to determine the value of $k$ from an experiment. Nonetheless, it has been observed experimentally that the leading front of the flashbacking, swirling flames are quite thin [48]. These evidence imply that most probably $r_{u}$ remains close to the radius of the bluff-body and therefore, in reality, the values of $\mathrm{k}$ and $K^{\prime}$ are expected to be close to each other. 


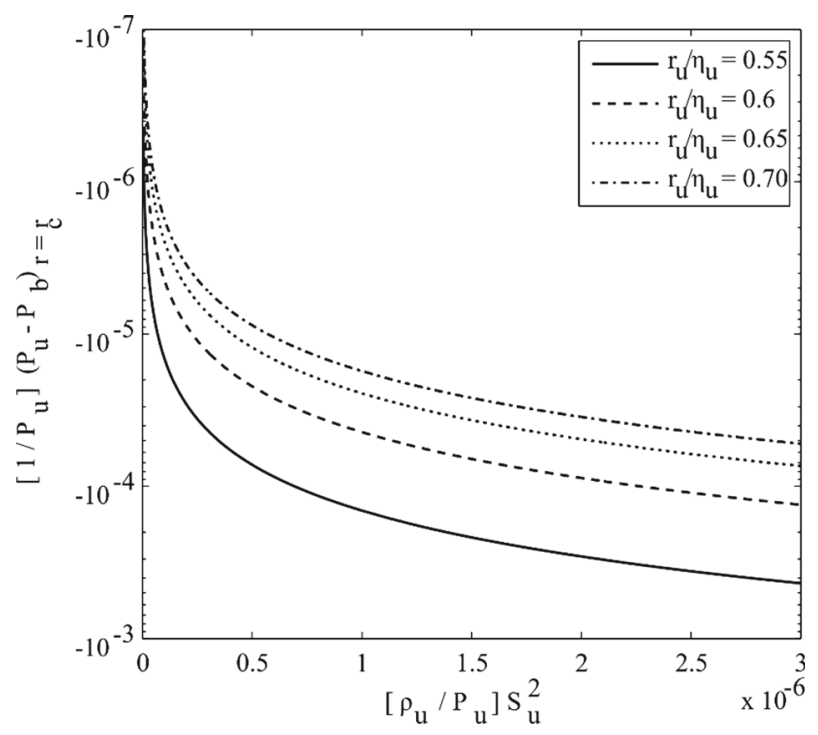

Fig. 9 Effect of altering the burning ratio on the pressure difference across the flame, for varying dimensionless flame speed

It has been shown in previous studies $[27,52]$ that in a swirling arrangement flame displacement velocity can go through significant changes. Figures 10 and 11 show the modifications of the flame angular, displacement velocity with variations in laminar flame speed, cold flow angular velocity (Fig. 10) and the expansion ratio (Fig. 11). The negative value of the displacement velocity in these figures is the result of the propagation direction considered in Fig. 4 and, is in agreement with the original theory of Ishizuka [27] and its later extension [52]. Both of these figures show that, expectedly, in the limit of zero laminar flame speed the angular flame velocity approaches zero. Further, displacement angular velocity appears to correlate with the laminar flame speed. Furthermore, faster cold flows in Fig. 10 and larger expansion ratios in Fig. 11 result in higher angular displacement velocities. The latter is consistent with the discussion on Fig. 7. The back pressure effects, including the pressure difference and enhancement of the flame displacement speed, are sensitive to expansion ratio and are, generally, signified by increasing this parameter.

It is interesting to note that the qualitative behaviours in Figs. 5, 6, 7, 8, 9, 10 and 11 are in total agreement with those in the corresponding case of axially propagating swirling flame [52]. This similarity seems plausible as the fundamental mechanism of back pressure and flame displacement enhancing are the same in these two different cases. The propagation direction determines the direction of the momentum flow across the flame. However, since in both axial and circumferential configurations the flame propagates against the cold flow, it features the same mechanism of pressure gain. That is to maintain the flow rotation, the light hot gas downstream of the reactive front demands a smaller centrifugal force compared to the heavy, cold gas upstream of the flame. As explained by Ishizuka [27], this causes a positive pressure gradient across the flame. It is, further, worth noting that, although not shown here, the observed qualitative trends are independent of the choice of the vortex model. The most important feature of the back pressure effect is the change in the momentum flow due to radical density jumps across a swirling flame [27]. Hence, provided that the 


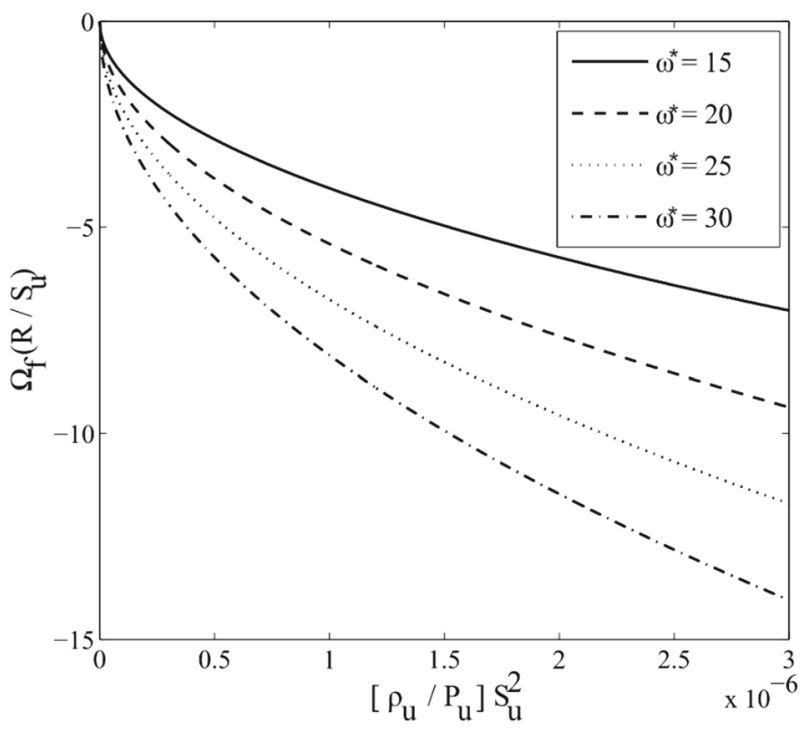

Fig. 10 The effect of changing the upstream angular velocity on the flames angular velocity for varying dimensionless flame speed

density changes and the total circumferential momentum in the burnt and unburnt regions remain unchanged, any vortex model is expected to result in the pressure changes similar to those calculated here.

An inspection of Figs. 5, 6, 7, 8, 9, 10 and 11 reveals that for a stoichiometric air and methane mixture and configuration similar to the experimental setup (Fig. 1) the theory predicts a pressure gain of order $10 \mathrm{~Pa}$. This is within the same order of magnitude as the experimentally measured pressure gain shown in Fig. 2. The previous theoretical analysis on a purely axially propagating, rotational flame, also predicted the same order of magnitude for the pressure gain across the flame [52]. It is important to note that the experimentally observed flame flashback includes both axial and circumferential propagation of the flame. The premixed turbulent flame in the experiment features a swirl number close to unity. Hence, as supported by the theoretical results, the axial and circumferential effects are expected to have comparable significance. As discussed previously [52], given the relative simplicity of the theory and sophistication of the real reactive flow, the similarities between the theoretical predictions and experiment is striking. In particular, the presence of the strongly turbulent flow is anticipated to make a significant distinction between the measured values and the predictions of the current laminar theory. Nevertheless, the observed agreement between the theory and experiment strengthens the speculation that the leading propagation front, in the experiment, could be in laminar regime. It is important to note that direct numerical simulations, in other configurations, have confirmed the closeness of the leading flashback front to the wall [21]. However, currently, there exist limited experimental and numerical data on the state of the boundary layer in this configuration [48, 49]. These data are insufficient to clarify the regime of the boundary layer ahead of the flame. Nonetheless, they indicate the existence of a thickened or separated boundary layer ahead of the flashbacking front $[48,49]$. 


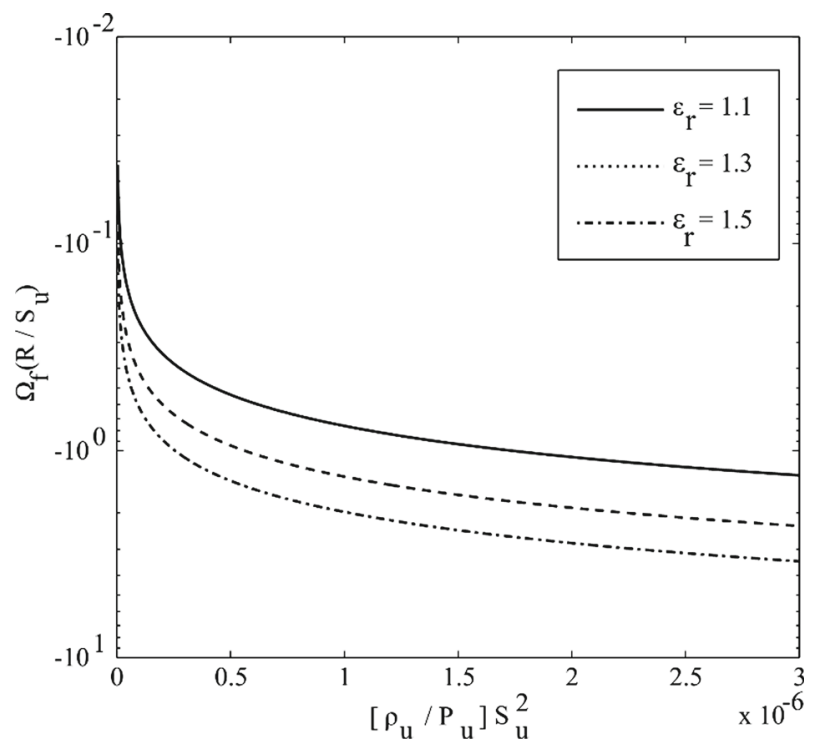

Fig. 11 Effect of variations in expansion ratio on the flames angular velocity for varying dimensionless flame speed

A comprehensive explanation of flame flashback in the configuration shown in Fig. 1 is yet to be developed. In the meantime, experimental [48] and numerical studies [49] indicate that there are, at least, two routes to flashback in this system. First, vorticity generation, primarily by baroclinic torque, forms a recirculation zone and induces a negative velocity ahead of the flame [49]. Second, the boundary layer on the surface of the bluff-body goes through a thickening or separation process and the flame propagates upstream in the generated low velocity region [48]. The first route has been also observed in other configurations [39]. The relative significance of these two is, currently, unclear. Nonetheless, regardless of this uncertainty about the mechanism of flashback, the back pressure can have important effects in the series events constituting flame flashback. The positive pressure gradient can significantly influence the baroclinic torque and therefore affect the induction of negative velocity. This has been already regarded as the physical mechanism for production of the recirculation zone ahead of the flame [49]. Further, formation of an axial adverse pressure gradient was noted in the work of Heeger et al. [48] as the element of reactive flow which drives the boundary layer towards separation. Most importantly, the arguments in both of these studies [48, 49] remained mostly heuristic and lacked a rigorous theoretical treatment. The current analysis and results, along with the previous ones on axial propagation [52], provide an insight into the physics of pressure gain across the flame. As stated earlier, this is the main aim of this work.

Finally, it is important to note that in order to make a more comprehensive theory the results of the circumferential and axial analyses cannot be merely added to each other. This is due to the fact that none of these are linear. These theories are only meant to describe parts of the physical mechanisms active during the upstream flame propagation. Extension of the current theoretical analysis to the cases including both circumferential and axial flame propagation is an outstanding future task. 


\section{Conclusions}

Upstream circumferential propagation of a premixed, laminar flame was analysed theoretically. Through extension of the original theory of flame back pressure [27], an analytical model was developed to predict the generation of adverse pressure gradient across the flame. Enhancement of flame displacement velocities was also calculated. It was argued that the change of centrifugal forces on the two sides of the flame is responsible for the pressure gain in the circumferentially propagating flame. This mechanism is on the basis of the back pressure theory [27] and remains essentially indifferent by changing the propagation direction from axial to circumferential. The results were in agreement with an earlier extension of the original theory, which considers axial propagation of a rotating flame [52]. More importantly, they were consistent with the experimental measurements of the pressure across the flame. These confirmed that in reality the developed flame back pressure features contributions from both axial and circumferential propagations. The relative significance of these two is expected to depend upon the flow swirl number. For those swirl numbers pertinent to practical flashbacking flames they appeared to be of similar importance. It was argued that the developed adverse pressure gradient can affect the boundary layer on the surface of the bluff-body and thicken or possibly separate that. Further, the higher pressure in the burned gas may contribute with the baroclinic torque and strengthen the vorticity generation which in turn leads to the production of negative velocity.

In interpreting the results of this study, it is essential to note that the emerging physical picture of flame flashback, in general, appears to be immensely complicated [21, 44]. Multiple physical processes, including vorticity generation [38-43, 49], aerodynamically induced back pressure [27, 28, 44], flame wall interactions [16-21], coherent structures [47, 48], flame stretch and heat release fluctuations $[42,45]$ have been identified as highly influential effects. The configuration analysed in the experimental part of this paper, features flashback in the core flow and also involves strong flame wall interaction [47, 48, 51]. It is, therefore, unlikely that a single mechanism can be regarded as the driving force of flashback in this configuration. The observed flashback is, most probably, the net result of multiple, interacting mechanisms. Hence, an ultimate understanding of the observed flashback is subject to understanding all the influential effects. The current theoretical results showed that, as speculated previously $[48,49]$, there exists a pressure force which can significantly affect the flow field and flame flashback. Indeed, the interactions of the detected adverse pressure gradient with the reactive flow may lead to more complex processes, which remain to be analysed in future.

\section{Nomenclature}

$\begin{array}{ll}i & \text { Imaginary number } \equiv \sqrt{-1} \\ k & \text { Burning ratio } \equiv \frac{r_{u}}{\eta_{u}} \\ l & \text { Length of combustor } \\ \mathbf{n} & \text { Normal unit vector } \\ r & \text { Radial coordinate } \\ r_{b} & \text { Radius of burned mixture downstream of the flame (m) } \\ r_{c} & \text { Radius of the bluff body (m) } \\ r_{u} & \text { Radius of the unburned mixture downstream of the flame that later burns in the } \\ u & \text { flame }(\mathrm{m}) \\ & \text { Velocity }(\mathrm{m} / \mathrm{s})\end{array}$


A Area $\left(\mathrm{m}^{2}\right)$

$K \quad$ Radius of the forced vortex core upstream of the flame to the radius of the cylindrical tube $\equiv \frac{\eta_{u}}{R}$

$K^{\prime} \quad$ Ratio of the radius of the bluff body to the radius of the forced vortex core upstream of the flame $\equiv \frac{r_{c}}{\eta_{u}}$

$P \quad$ Pressure (Pa)

$P_{b} \quad$ Pressure in the burned region (Pa)

$P_{b}(r) \quad$ Downstream pressure measured at the radial distance $r(\mathrm{~Pa})$

$P_{u} \quad$ Pressure in the unburned region $(\mathrm{Pa})$

$P_{u}(r) \quad$ Upstream pressure measured at the radial distance $\mathrm{r}(\mathrm{Pa})$

$R \quad$ Radius of the cylindrical tube (combustor radius) (m)

$S_{u} \quad$ Laminar burning velocity $(\mathrm{m} / \mathrm{s})$

$S_{u}^{*} \quad$ Non-dimensional laminar burning velocity $(\mathrm{m} / \mathrm{s})$

$T \quad$ Temperature $(\mathrm{K})$

V Velocity vector $(\mathrm{m} / \mathrm{s})$

$V_{b} \quad$ Axial velocity of the burned mixture downstream of the flame in the forced vortex core $(\mathrm{m} / \mathrm{s})$

$V_{f} \quad$ Flame velocity $(\mathrm{m} / \mathrm{s})$

$V_{r} \quad$ Relative velocity $(\mathrm{m} / \mathrm{s})$

$V_{u} \quad$ Axial velocity of unburned mixture upstream of the flame $(\mathrm{m} / \mathrm{s})$

$V_{z} \quad$ Axial velocity $(\mathrm{m} / \mathrm{s})$

$V_{\theta} \quad$ Tangential velocity $(\mathrm{m} / \mathrm{s})$

$V_{\theta_{\max }} \quad$ Maximum tangential velocity $(\mathrm{m} / \mathrm{s})$

$V_{\theta_{r}} \quad$ Circumferential velocity in the rotating coordinate $(\mathrm{m} / \mathrm{s})$

$\alpha \quad$ Constant to simplify Eq.33

$\beta \quad$ Constant to simplify Eq.34

$\delta \quad$ Density ratio

$\Delta P \quad$ Change in pressure $(\mathrm{Pa})$

$\Delta P_{b} \quad$ Pressure change downstream of the flame (Pa)

$\Delta P_{u} \quad$ Pressure change upstream of the flame (Pa)

$(\Delta P)_{r=r_{c}} \quad$ Pressure difference across the flame at the wall of the bluff body (Pa)

$\epsilon_{r} \quad$ Expansion ratio $\equiv r_{b} / r_{u}$

$\epsilon_{r}^{\prime} \quad$ Ratio of the downstream to upstream forced vortex radii $\equiv \eta_{u}^{\prime} / \eta_{u}$

$\epsilon_{r}^{\prime} \quad$ Expansion ratio amended for the presence of the bluff body

$\eta_{u} \quad$ Radius of the forced vortex core upstream of the flame (m)

$\eta_{u}^{\prime} \quad$ Radius of the forced vortex core downstream of the flame (m)

$\mu \quad$ Viscosity $\mathrm{kg} /(\mathrm{s} . \mathrm{m})$

$\rho \quad$ Density $\left(\mathrm{kg} / \mathrm{m}^{3}\right)$

$\rho_{b} \quad$ Density of the burned gas $\left(\mathrm{kg} / \mathrm{m}^{3}\right)$

$\rho_{u} \quad$ Density of the unburned gas $\left(\mathrm{kg} / \mathrm{m}^{3}\right)$

$\varphi \quad$ Equivalence ratio

$\omega^{*} \quad$ Non-dimensional upstream angular velocity

$\Omega \quad$ Angular velocity (1/s)

$\Omega_{b} \quad$ Angular velocity of the burned mixture downstream of the flame (1/s)

$\Omega_{u} \quad$ Angular velocity of the unburned mixture upstream of the flame (1/s)

$\Omega_{u}^{\prime} \quad$ Angular velocity of the unburned mixture downstream of the flame (1/s) 
Acknowledgments The authors are grateful for the financial support by Deutsche Forschungsgemeinschaft through project KA 3483/1-1.

Open Access This article is distributed under the terms of the Creative Commons Attribution 4.0 International License (http://creativecommons.org/licenses/by/4.0/), which permits unrestricted use, distribution, and reproduction in any medium, provided you give appropriate credit to the original author(s) and the source, provide a link to the Creative Commons license, and indicate if changes were made.

\section{Appendix A: Series expansion}

Expanding the expression $\ln \left(\epsilon_{r}^{\prime}\right)$ to the orders on the right hand side of Eq. A-1 provides a systematically more accurate value for $\epsilon_{\mathrm{r}}$. These are presented in the followings. It is known that [53]

$$
\ln (x)=\sum_{n=1}^{\infty} \frac{(-1)^{n}(x-1)^{n}}{n}=(x-1)-\frac{(x-1)^{2}}{2}+\frac{(x-1)^{3}}{3}-\frac{(x-1)^{4}}{4}
$$

Expansion of order 1

$$
\begin{array}{r}
\ln \left(\epsilon_{r}^{\prime}\right)=\left(\epsilon_{r}^{\prime}-1\right) \\
\left(\epsilon_{r}^{\prime}-1\right)\left[1-k^{2}-\epsilon_{r}^{\prime 2}+\left(\epsilon_{r} k\right)^{2}\right]=2 k^{2}\left[\epsilon_{r}^{2}-\left(\frac{\epsilon_{r}}{\epsilon_{r}^{\prime}}\right)^{2}-\epsilon_{r}^{\prime 2}+1\right]
\end{array}
$$

Expanding the brackets and multiplying by $\epsilon_{r}^{\prime 2}$ to cancel of the quadratic denominator on the right hand side of the equation produces:

$$
\begin{aligned}
\epsilon_{r}^{\prime 5}(-1)+ & \epsilon_{r}^{\prime 4}\left(1+2 k^{2}\right)+\epsilon_{r}^{\prime 3}\left(1-k^{2}+\left(\epsilon_{r} k\right)^{2}\right)+\epsilon_{r}^{\prime 2}\left(-1-k^{2}+\left(\epsilon_{r} k\right)^{2}\right) \\
& +\epsilon_{r}^{\prime 1}(0)+\epsilon_{r}^{\prime 0}\left(2\left(\epsilon_{r} k\right)^{2}\right)=0
\end{aligned}
$$

Expansion of order 2,

$$
\begin{array}{r}
\ln \left(\epsilon_{r}^{\prime}\right)=\left(\epsilon_{r}^{\prime}-1\right)-\frac{\left(\epsilon_{r}^{\prime}-1\right)^{2}}{2}=\frac{\left(4 \epsilon_{r}^{\prime}-3-\epsilon_{r}^{\prime 2}\right)}{2} \\
\left(4 \epsilon_{r}^{\prime}-3-\epsilon_{r}^{\prime 2}\right)\left[1-k^{2}-\epsilon_{r}^{\prime 2}+\left(\epsilon_{r} k\right)^{2}\right]=4 k^{2}\left[\epsilon_{r}^{2}-\left(\frac{\epsilon_{r}}{\epsilon_{r}^{\prime}}\right)^{2}-\epsilon_{r}^{\prime 2}+1\right]
\end{array}
$$

By means of the same procedure

$$
\begin{aligned}
\epsilon_{r}^{\prime 6}(1)+ & \epsilon_{r}^{\prime 5}(-1)+\epsilon_{r}^{\prime 4}\left(2+5 k^{2}-\left(\epsilon_{r} k\right)^{2}\right)+\epsilon_{r}^{\prime 3}\left(4-4 k^{2}+\left(\epsilon_{r} k\right)^{2}\right) \\
& +\epsilon_{r}^{\prime 2}\left(-3-k^{2}-7\left(\epsilon_{r} k\right)^{2}\right)+\epsilon_{r}^{\prime 1}(0)+\epsilon_{r}^{\prime 0}\left(4\left(\epsilon_{r} k\right)^{2}\right)=0
\end{aligned}
$$


Expansion of order 3:

$$
\begin{aligned}
& \ln \left(\epsilon_{r}^{\prime}\right)=\left(\epsilon_{r}^{\prime}-1\right)-\frac{\left(\epsilon_{r}^{\prime}-1\right)^{2}}{2}+\frac{\left(\epsilon_{r}^{\prime}-1\right)^{3}}{3}=\frac{2 \epsilon_{r}^{\prime 3}-9 \epsilon_{r}^{\prime 2}+18 \epsilon_{r}^{\prime}-11}{6}, \\
&\left(2 \epsilon_{r}^{\prime 3}-9 \epsilon_{r}^{\prime 2}+18 \epsilon_{r}^{\prime}-11\right)\left[1-k^{2}-\epsilon_{r}^{\prime 2}+\left(\epsilon_{r} k\right)^{2}\right]=12 k^{2}\left[\epsilon_{r}^{2}-\left(\frac{\epsilon_{r}}{\epsilon_{r}^{\prime}}\right)^{2}-\epsilon_{r}^{\prime 2}+1\right] . \\
& \epsilon_{r}^{\prime 7}(-2)+\epsilon_{r}^{\prime 6}(9)+\epsilon_{r}^{\prime 5}\left(-16-k^{2}+2\left(\epsilon_{r} k\right)^{2}\right)+\epsilon_{r}^{\prime 4}\left(2+21 k^{2}\right. \\
&\left.-9\left(\epsilon_{r} k\right)^{2}\right)+\epsilon_{r}^{\prime 3}\left(18-18 k^{2}+18\left(\epsilon_{r} k\right)^{2}\right)+\epsilon_{r}^{\prime 2}\left(-11-k^{2}\right. \\
&\left.-23\left(\epsilon_{r} k\right)^{2}\right)+\epsilon_{r}^{\prime 1}(0)+\epsilon_{r}^{\prime 0}\left(12\left(\epsilon_{r} k\right)^{2}\right)=0
\end{aligned}
$$

Expansion of order 4:

$$
\begin{aligned}
& \ln \left(\epsilon_{r}^{\prime}\right)=\left(\epsilon_{r}^{\prime}-1\right)-\frac{\left(\epsilon_{r}^{\prime}-1\right)^{2}}{2}+\frac{\left(\epsilon_{r}^{\prime}-1\right)^{3}}{3}-\frac{\left(\epsilon_{r}^{\prime}-1\right)^{4}}{4}=\frac{3 \epsilon_{r}^{\prime 4}-8 \epsilon_{r}^{\prime 3}+24 \epsilon_{r}^{\prime}-19}{12}, \\
&\left(3 \epsilon_{r}^{\prime 4}-8 \epsilon_{r}^{\prime 3}+24 \epsilon_{r}^{\prime}-19\right)\left[1-k^{2}-\epsilon_{r}^{\prime 2}+\left(\epsilon_{r} k\right)^{2}\right]=24 k^{2}\left[\epsilon_{r}^{2}-\left(\frac{\epsilon_{r}}{\epsilon_{r}^{\prime}}\right)^{2}-\epsilon_{r}^{\prime 2}+1\right] . \\
& \epsilon_{r}^{\prime 8}(-3)+\epsilon_{r}^{\prime 7}(8)+\epsilon_{r}^{\prime 6}\left(3-3 k^{2}+3\left(\epsilon_{r} k\right)^{2}\right)+\epsilon_{r}^{\prime 5}\left(-32+8 k^{2}-8\left(\epsilon_{r} k\right)^{2}\right) \\
&+\epsilon_{r}^{\prime 4}\left(19+24 k^{2}\right)+\epsilon_{r}^{\prime 3}\left(24-24 k^{2}+24\left(\epsilon_{r} k\right)^{2}\right) \\
&+\epsilon_{r}^{\prime 2}\left(-19-5 k^{2}-43\left(\epsilon_{r} k\right)^{2}\right)+\epsilon_{r}^{\prime 1}(0)+\epsilon_{r}^{\prime 0}\left(24 k^{2}\right)=0 .
\end{aligned}
$$

To find an accurate approximation for $\epsilon_{r}^{\prime}$ the roots of the fourth order polynomial (A-5) are found numerically.

\section{References}

1. Aguilar, F.J.E., Quintero, R.R., Trujillo, E.C., García, M.T.: Analysis of regulation methods of a combined heat and power plant based on gas turbines. Energy 72, 574-589 (2014)

2. Taamallah, S., Vogiatzaki, K., Alzahrani, F.M., Mokheimer, E.M.A., Habib, M.A., Ghoniem, A.F.: Fuel flexibility, stability and emissions in premixed hydrogen-rich gas turbine combustion: Technology, fundamentals, and numerical simulations. Appl. Energ. 154, 1020-1047 (2015)

3. Chaos, M., Dryer, F.L.: Syngas combustion kinetics and applications. Combust. Sci. Technol. 180(6), 1053-1096 (2008)

4. Di Sarli, V., Di Benedetto, A.: Laminar burning velocity of hydrogen-methane/air premixed flames. Int. J. Hydrogen Energ. 32(5), 637-646 (2007)

5. Wang, J., Huang, Z., Tang, C., Miao, H., Wang, X.: Numerical study of the effect of hydrogen addition on methane-air mixtures combustion. Int. J. Hydrogen Energ. 34(2), 1084-1096 (2009)

6. Lieuwen, T., McDonell, V., Petersen, E., Santavicca, D.: Fuel flexibility influences on premixed combustor blowout, flashback, autoignition, and stability. J. Eng. Gas Turb. Power 130(1), 011506 (2008)

7. Karimi, N.: Response of a conical, laminar premixed flame to low amplitude acoustic forcing-A comparison between experiment and kinematic theories. Energy 78, 490-500 (2014)

8. Tuncer, O., Acharya, S., Uhm, J.H.: Dynamics, NOx and flashback characteristics of confined premixed hydrogen-enriched methane flames. Int. J. Hydrogen Energ. 34(1), 496-506 (2009)

9. Syred, N., Abdulsada, M., Griffiths, A., O’Doherty, T., Bowen, P.: The effect of hydrogen containing fuel blends upon flashback in swirl burners. Appl. Energ. 89(1), 106-110 (2012)

10. Syred, N., Giles, A., Lewis, J., Abdulsada, M., Medina, A.V., Marsh, R., Griffiths, A.J.: Effect of inlet and outlet configurations on blow-off and flashback with premixed combustion for methane and a high hydrogen content fuel in a generic swirl burner. Appl. Energ. 116, 288-296 (2014) 
11. Khalil, A.E., Gupta, A.K.: Hydrogen addition effects on high intensity distributed combustion. Appl. Energ. 104, 71-78 (2013)

12. Jejurkar, S.Y., Mishra, D.P.: Flame stability studies in a hydrogen-air premixed flame annular microcombustor. Int. J. Hydrogen Energ. 36(12), 7326-7338 (2011)

13. Wierzba, I., Oladipo, A.B., Karim, G.A.: The limits for flame flashback within streams of lean homogeneous fuel-diluent-air mixtures involving hydrogen. Int. J. Hydrogen Energ. 18(3), 223-226 (1993)

14. Galley, D., Ducruix, S., Lacas, F., Veynante, D.: Mixing and stabilization study of a partially premixed swirling flame using laser induced fluorescence. Combust. Flame 158(1), 155-171 (2011)

15. Lewis, B., von Elbe, G.: Combustion, Flames and Explosion of Gases. Academic, New York (1951)

16. Kurdyumov, V.N., Fernández, E., Linan, A.: Flame flashback and propagation of premixed flames near a wall. Proceed. Combust. Inst. 28(2), 1883-1889 (2000)

17. Kurdyumov, V., Fernandez-Tarrazo, E., Truffaut, J.M., Quinard, J., Wangher, A., Searby, G.: Experimental and numerical study of premixed flame flashback. Proceed. Combust. Inst. 31(1), 1275-1282 (2007)

18. Eichler, C., Sattelmayer, T.: Experiments on flame flashback in a quasi-2D turbulent wall boundary layer for premixed methane-hydrogen-air mixtures. J. Eng. Gas Turb. Power 133(1), 011503 (2011)

19. Eichler, C., Sattelmayer, T.: Premixed flame flashback in wall boundary layers studied by long-distance micro-PIV. Exp. Fluids 52(2), 347-360 (2012)

20. Eichler, C., Baumgartner, G., Sattelmayer, T.: Experimental investigation of turbulent boundary layer flashback limits for premixed hydrogen-air flames confined in ducts. J. Eng. Gas Turb. 134(1), 011502 (2012)

21. Gruber, A., Chen, J.H., Valiev, D., Law, C.K.: Direct numerical simulation of premixed flame boundary layer flashback in turbulent channel flow. J. Fluid Mech. 709, 516-542 (2012)

22. Huang, Y., Yang, V.: Effect of swirl on combustion dynamics in a lean-premixed swirl-stabilized combustor. Proc. Combust. Inst. 30(2), 1775-1782 (2005)

23. Dam, B., Corona, G., Hayder, M., Choudhuri, A.: Effects of syngas composition on combustion induced vortex breakdown (CIVB) flashback in a swirl stabilized combustor. Fuel 90(11), 3274-3284 (2011)

24. Dam, B., Love, N., Choudhuri, A.: Flashback propensity of syngas fuels. Fuel 90(2), 618-625 (2011)

25. Chomiak, J.: Dissipation fluctuations and the structure and propagation of turbulent flames in premixed gases at high Reynolds numbers. In: Symposium (International) on combustion. Elsevier, vol. 16, pp. 1665-1673 (1977)

26. McCormack, P.D., Scheller, K., Mueller, G., Tisher, R.: Flame propagation in a vortex core. Combust. Flame 19(2), 297-303 (1972)

27. Ishizuka, S.: Flame propagation along a vortex axis. Progr. Energ. Combust. Sci. 28(6), 477-542 (2002)

28. Ishizuka, S., Hamasaki, T., Koumura, K., Hasegawa, R.: Measurements of flame speeds in combustible vortex rings: validity of the back-pressure drive flame propagation mechanism. In: Symposium (International) on combustion. Elsevier, vol. 27, pp. 727-734 (1998)

29. Poinsot, T., Veynante, D.: Theoretical and numerical combustion, 2nd edn. Edwarsd, Inc, Philadelphia (2005)

30. Atobiloye, R.Z., Britter, R.E.: On flame propagation along vortex tubes. Combust. Flame 98(3), 220230 (1994)

31. Sakai, Y.: The phenomena of flame propagation in a rotating tube. In: Symposium (International) on combustion, vol. 26, pp. 847-853 (1996)

32. Ishizuka, S., Koumura, K., Hasegawa, R.: Enhancement of flame speed in vortex rings of rich hydrogen/air mixtures in air. Proc. Combust. Institute 28(2), 1949-1956 (2000)

33. Lucca-Negro, O., O'doherty, T.: Vortex breakdown: a review. Prog. Energ. Combust. Sci. 27(4), 431481 (2001)

34. Escudier, M.: Vortex breakdown: observations and explanations. Prog. Aerospace Sci. 25(2), 189-229 (1988)

35. Leibovich, S.: The structure of vortex breakdown. Annu. Rev. Fluid Mech. 10(1), 221-246 (1978)

36. Fritz, J., Kroner, M., Sattelmayer, T.: Flashback in a swirl burner with cylindrical premixing zone. J. Eng. Gas Turb. Power 126(2), 276-283 (2004)

37. Kroner, M., Fritz, J., Satelmayer, T.: Flashback limits for combustion induced vortex breakdown in a swirl burner. J. Eng. Gas Turb. Power 125(3), 693-700 (2003)

38. Konle, M., Kiesewetter, F., Sattelmayer, T.: Simultaneous high repetition rate PIV-LIF-measurements of CIVB driven flashback. Exp. Fluids 44(4), 529-538 (2008)

39. Kiesewetter, F., Konle, M., Sattelmayer, T.: Analysis of combustion induced vortex breakdown driven flame flashback in a premix burner with cylindrical mixing zone. J. Eng. Gas Turb. Power 129(4), 929936 (2007) 
40. Kröner, M., Sattelmayer, T., Fritz, J., Kiesewetter, F., Hirsch, C.: Flame propagation in swirling flowseffect of local extinction on the combustion induced vortex breakdown. Combust. Sci. Technol. 179(7), 1385-1416 (2007)

41. Voigt, T., Habisreuther, P., Zarzalis, N.: Simulation of vorticity driven flame instability using a flame surface density approach including Markstein number effects. In: ASME turbo expo 2009: power for land, sea, and air, pp. 255-264. American society of mechanical engineers (2009)

42. Konle, M., Sattelmayer, T.: Time scale model for the prediction of the onset of flame flashback driven by combustion induced vortex breakdown. J. Eng. Gas Turb. Power 132(4), 041503 (2010)

43. Blesinger, G., Koch, R., Bauer, H.J.: Influence of flow field scaling on flashback of swirl flames. Exp. Therm. Fluid Sci. 34(3), 290-298 (2010)

44. Lieuwen, T.C.: Unsteady combustor physics. Cambridge University Press (2012)

45. Baumgartner, G., Sattelmayer, T.: Experimental investigation of the flashback limits and flame propagation mechanisms for premixed hydrogen-air flames in non-swirling and swirling flow. In: ASME turbo expo 2013: turbine technical conference and exposition, pp. V01AT04A010-V01AT04A010. American Society of Mechanical Engineers (2013)

46. Sattelmayer, T., Mayer, C., Sangl, J.: Interaction of flame flashback mechanisms in premixed hydrogen-air swirl flames. In: ASME turbo expo 2014: turbine technical conference and exposition, pp. V04AT04A041-V04AT04A041. American Society of Mechanical Engineers (2014)

47. Nauert, A., Petersson, P., Linne, M., Dreizler, A.: Experimental analysis of flashback in lean premixed swirling flames: conditions close to flashback. Exp. Fluids 43(1), 89-100 (2007)

48. Heeger, C., Gordon, R.L., Tummers, M.J., Sattelmayer, T., Dreizler, A.: Experimental analysis of flashback in lean premixed swirling flames: upstream flame propagation. Exp. Fluids 49(4), 853-863 (2010)

49. De, A., Acharya, S.: Parametric study of upstream flame propagation in hydrogen-enriched premixed combustion: effects of swirl, geometry and premixedness. Int J. Hydrogen Energy 37(19), 14649-14668 (2012)

50. De, A., Acharya, S.: Dynamics of upstream flame propagation in a hydrogen-enriched premixed flame. Int. J. Hydrog. Energy 37(22), 17294-17309 (2012)

51. Schneider, C., Dreizler, A., Janicka, J.: Fluid dynamical analysis of atmospheric reacting and isothermal swirling flows. Flow, Turbul. Combust. 74(1), 103-127 (2005)

52. Karimi, N., Heeger, C., Christodoulou, L., Dreizler, A.: Experimental and theoretical investigation of the flashback of a swirling, bluff-body stabilised, premixed. Flame. Z. Phys. Chem. 229(5), 663-689 (2015)

53. Thomas, G.B., Finney, R.L.: Calculus and analytic geometry. Addison Wesley, New York (1992) 